\title{
Protocolo clínico de abordagem das reações agudas de hipersensibilidade a meios de contraste em Imagiologia
}

\author{
Clinical protocol of management of hypersensitivity reactions \\ to contrast media in Radiology
}

Rev Port Imunoalergologia $2019 ; 27$ (I): 4 I-54

Cristina Lopes ${ }^{1,2}$, Catarina Tavares ${ }^{3}$, Inês Rolla ${ }^{4}$, Ernestina Gomes ${ }^{5}$

Todos os autores contribuíram de igual forma para a elaboração do manuscrito

I Assistente Hospitalar Graduada. Unidade de Imunoalergologologia, Unidade Local de Saúde Matosinhos

${ }^{2}$ Professora Auxiliar. Laboratório de Imunologia Básica e Clínica, Faculdade de Medicina da Universidade do Porto

${ }^{3}$ Assistente Hospitalar Graduada. Serviço de Imagiologia, Unidade Local de Saúde, Matosinhos

${ }^{4}$ Interna Formação Específica. Serviço de Imagiologia, Unidade Local de Saúde, Matosinhos

${ }^{5}$ Assistente Hospitalar Graduada. Serviço de Medicina Intensiva Unidade Local de Saúde, Matosinhos, Coordenadora

de Emergência Interna da Unidade Local de Saúde, Matosinhos

\section{RESUMO}

As reações agudas de hipersensibilidade a meios de contraste (RAHC) em imagiologia são consideradas pouco frequentes; são habitualmente ligeiras, mas podem ser potencialmente fatais. Apesar de existirem recomendações nacionais e internacionais acerca da sua abordagem, não existe um protocolo clínico que integre a visão de todas as especialidades envolvidas (Imagiologia, Imunoalergologia e Medicina Intensiva). Pretendeu-se estabelecer um protocolo prático de utilização transversal e adequada à realidade hospitalar que inclua a definição das reações adversas e sua classificação, a identificação de fatores de risco para a ocorrência de reação de hipersensibilidade, a abordagem diagnóstica e terapêutica e de orientação dos doentes com RAHC. Realizou-se uma revisão não sistemática das recomendações nacionais e internacionais acerca da abordagem das RAHC publicadas pelas sociedades científicas de Imagiologia, Imunoalergologia e Medicina Intensiva. Elaborou-se um protocolo por consenso de peritos destas especialidades.

Palavras-chave: meios de contraste, hipersensibilidade, reação adversa, imagiologia. 


\section{ABSTRACT}

Hypersensitivity reactions to contrast media (HSR) are infrequent, usually mild but potentially fatal. Although there are recommendations from Radiology, Intensive care and Allergology fields regarding its management, a clinical multidisciplinary protocol that integrates these complementary approaches is missing. We aimed to elaborate a protocol with adverse reactions definition, classification and risk factors identification and management of patients with HSR. A non-systematic revision of national and international guidelines was made regarding the management of hypersensitivity reactions, in order to elaborate a clinical consensus protocol to be used in different medical fields (Radiology, Allergology and Intensive care).

Key-words: Contrast medium, hypersensitivity, adverse reaction, radiology.

\section{INTRODUÇÃO}

A s reações adversas agudas não nefrotóxicas com meio de contraste ocorrem em cerca de $0,2 \%$ a $0,7 \%$ das administrações com contrastes iodados e em 0,24\% a 0,07\% com gadolínio, sendo as reações graves ainda mais raras ${ }^{1,2}$. Apesar de pouco frequentes, estas reações podem ser fatais, sendo necessário identificar fatores de risco prévios à administração de meios de contraste e estabelecer um algoritmo diagnóstico e terapêutico adequados.

\section{OBJETIVOS}

- Abordar a definição de reação de hipersensibilidade a meios de contraste estabelecendo o diagnóstico diferencial com reações quimiotóxicas;

- Determinar os fatores de risco para reações de hipersensibilidade;

- Estabelecer os procedimentos laboratoriais a realizar em caso de suspeita de reação alérgica;

- Uniformizar critérios diagnósticos clínicos e de tratamento das reações de hipersensibilidade;

- Definir critérios de orientação para a consulta de Imunoalergologia.
As reações adversas tardias (entre uma hora até vários dias após a administração de meio de contraste) e as reações nefrotóxicas a meios de contraste em imagiologia estão fora do âmbito deste protocolo.

Este protocolo poderá ser aplicado a todos os utentes submetidos a exames imagiológicos com administração de meios de contraste, podendo fazer parte das boas práticas associadas à utilização de meios de contraste em qualquer Serviço de Imagiologia.

Em imagiologia são utilizados vários meios de contraste e várias vias de administração. Pela frequência e significado clínico das reações adversas associadas à utilização dos meios de contraste, este protocolo refere-se apenas aos meios de contraste com iodo e com gadolínio, quando administrados por via endovenosa ou intra-arterial.

\section{DEFINIÇÕES}

Define-se uma reação de hipersensibilidade a meio de contraste como uma reação nociva e não intencional que ocorre até uma hora depois da administração do meio de contraste e que não decorre do efeito nefrotóxico do mesmo'.

Estas reações podem ser observadas com meios de contraste iodados em procedimentos como angiografia, 
urografia e tomografia computorizada e com meios de contraste com gadolínio na realização de ressonância magnética, sendo, no entanto, mais frequentes com os meios de contraste iodados ${ }^{2}$. Em termos de gravidade, as mais frequentes habitualmente são ligeiras, enquanto situações ameaçadoras da vida são raras.

As reações adversas agudas não nefrotóxicas podem ser subdivididas em reações quimiotóxicas e reações de hipersensibilidade ${ }^{2}$.

\section{As reações quimiotóxicas:}

- Estão relacionadas com as propriedades químicas dos contrastes administrados em imagiologia, e são geralmente dependentes da dose e da taxa de infusão;

- São habitualmente transitórias e autolimitadas, mas podem ser graves. Incluem com relativa frequência a sensação de calor, rubor, náuseas e vómitos;

- As reações vasovagais são um tipo de reação quimiotóxica menos frequente, caracterizada por hipotensão e bradicardia;

- Devem ainda ser considerados os efeitos adversos cardiovasculares no contexto de reação quimiotóxica, como por exemplo arritmias, depressão da contratilidade cardíaca, crise hipertensiva e edema pulmonar cardiogénico. Em casos de hipotensão grave pode ocorrer perda de consciência, paragem cardiorrespiratória, angina ou convulsão. Estes efeitos são muitíssimo raros;

- A ocorrência destas reações não impede a administração de meio de contraste em futuros estudos de imagiologia.

\section{As reações de hipersensibilidade:}

- São idiossincráticas e imprevisíveis e podem ocorrer em resposta à administração de quantidades mínimas de meio de contraste;

- Podem ser alérgicas, mediadas por lgE / linfócitos T ou então mediadas por mecanismos inespecíficos de libertação de mediadores vasoativos (efeito direto na membrana do endotélio, ativação do sistema complemento ou formação direta de bradicininas).

\section{CLASSIFICAÇÃO DOSTIPOS DE CONTRASTES E GRAVIDADE DAS REAÇÕES ADVERSAS}

Os meios de contraste iodado $(\mathrm{MCl})$ são sais de iodo cuja estrutura química inclui um anel de benzeno com pelo menos três átomos de iodo que é responsável pela radiopacidade.

Os $\mathrm{MCl}$ podem ser classificados de acordo com carga da molécula iodada (iónicos e não iónicos), estrutura molecular (monoméricos e diméricos) e osmolaridade (hiperosmolar, baixa osmolaridade e iso-osmolaridade) ${ }^{2}$. A osmolaridade do contraste é o fator mais frequentemente associado a reações de hipersensibilidade sendo que as reações são mais frequentes com os de elevada osmolaridade.

A maioria dos contrastes classifica-se em:

- lónicos monoméricos, têm a maior osmolaridade, utilizam-se essencialmente em procedimentos extravasculares, como cistogramas;

- lónicos diméricos - o único exemplo é o ioxaglato e é de baixa osmolaridade;

- Não iónicos monoméricos, são considerados de segunda geração, têm baixa osmolaridade. Exemplos: iohexol, iopamidol, ioversol, iopromida, ioxilan, iomeprol;

- Não iónicos diméricos, exemplo: iodixanol. Está associado a igual ou menor número de reações de hipersensibilidade do que os de baixa osmolaridade.

Os meios de contraste utilizados na ressonância magnética são complexos de gadolínio que podem ser classificados em iónicos, não iónicos e, de acordo com a sua estrutura, em lineares ou macrocíclicos.

A classificação das reações adversas agudas a meios de contraste em imagiologia pode ser feita, de acordo com a gravidade clínica, em reações ligeiras, moderadas e graves $^{3}$ (Quadro I).

Em casos de reações cardiovasculares graves, como edema pulmonar, instabilidade hemodinâmica significativa e paragem cardiorrespiratória, embora estas reações possam resultar de uma reação quimiotóxica, pode ser 
Quadro I. Classificação das reações adversas de acordo com a gravidade

\begin{tabular}{|c|c|}
\hline Reações de hipersensibilidade & Reações quimiotóxicas \\
\hline \multicolumn{2}{|c|}{ Ligeiras } \\
\hline $\begin{array}{l}\text { Urticária / prurido limitados } \\
\text { Edema cutâneo limitado } \\
\text { Garganta "arranhada" } \\
\text { Congestão nasal } \\
\text { Espirros / conjuntivite / rinorreia }\end{array}$ & $\begin{array}{l}\text { Vómitos e náuseas limitados } \\
\text { Flushing /sensação de calor } \\
\text { Tremores transitórios } \\
\text { Cefaleia / tontura } \\
\text { Ansiedade } \\
\text { Alteração do paladar }\end{array}$ \\
\hline \multicolumn{2}{|c|}{ Moderadas } \\
\hline $\begin{array}{l}\text { Urticária / prurido generalizados } \\
\text { Eritema difuso com sinais vitais estáveis } \\
\text { Edema facial sem dispneia } \\
\text { Pieira / broncospasmo ligeiro }\end{array}$ & $\begin{array}{l}\text { Vómitos e náuseas intensos } \\
\text { Urgência hipertensiva } \\
\text { Dor torácica isolada } \\
\text { Reação vasovagal (que requer tratamento e responde ao } \\
\text { tratamento) }\end{array}$ \\
\hline \multicolumn{2}{|c|}{ Graves } \\
\hline $\begin{array}{l}\text { Edema facial ou generalizado com dispneia } \\
\text { Eritema difuso com hipotensão } \\
\text { Edema laríngeo com estridor e /ou hipóxia } \\
\text { Pieira/broncospasmo com hipóxia } \\
\text { Edema pulmonar não cardiogénico } \\
\text { Choque anafilático }\end{array}$ & $\begin{array}{l}\text { Reação vasovagal resistente } \\
\text { Arritmias } \\
\text { Convulsões } \\
\text { Emergência hipertensiva } \\
\text { Edema pulmonar cardiogénico }\end{array}$ \\
\hline
\end{tabular}

assumida uma etiologia alérgica para tratamento e referenciação futura do utente, sobretudo se coexistirem sinais e sintomas de envolvimento de pele e mucosas.

A prevalência de reações adversas agudas com meio de contraste iodado de baixa osmolaridade, a é muito baixa $(0,2 \%$ a $0,7 \%)$ e as reações graves são ainda mais raras $(0,04 \%)$. Com meio de contraste com gadolínio a prevalência de reações adversas agudas é muito mais baixa do que com contrastes iodados (variando entre $0,24 \%$ e $0,07 \%$ ), sendo que as reações graves alérgicas são muitíssimo raras (com prevalências de 0,01 a $0,001 \%)^{1,2}$.

\section{ABORDAGEM DOS DOENTES COM FATORES DE RISCO}

Podem ser considerados dois tipos de fatores de risco para reações de hipersensibilidade ${ }^{3,4}$

\section{Relativamente ao meio de contraste}

As reações são mais frequentes com os meios de contraste de elevada osmolaridade, no entanto, a prevalência de reações graves parece ser independente do grau de osmolaridade.

\section{Relativamente ao doente}

Os principais fatores de risco para reação de hipersensibilidade a meio de contraste são:

- História prévia de reação de hipersensibilidade em estudo de imagiologia com administração do meio de contraste;

- Asma instável.

Alguns autores consideram que a história prévia de asma brônquica, alergia a fármacos, alergia alimentar e sexo feminino podem constituir um fator de risco relativo, no entanto estes antecedentes não são impeditivos da sua realização nem determinam a necessidade de pré-medicação. 
Ressalva-se que a ocorrência de reação quimiotóxica prévia a meio de contraste endovenoso não constitui fator de risco para a ocorrência de reação de hipersensibilidade, pelo que não há lugar a pré-medicar estes doentes.

A abordagem do doente que vai realizar exame com meio de contraste é explicitada na Figura I.

\section{Atitudes a tomar em doentes com fatores de risco}

\section{Medidas gerais}

- Deve haver informação e coordenação recíproca entre a equipa de técnicos e de enfermeiros, e o médico neuro/radiologista;
- O doente deve permanecer no serviço de imagiologia, na área do TC / RM, com acesso venoso durante 30 minutos;

- Se for medicado com anti-histamínico com efeito sobre o estado de vigília (exemplo: clemastina IV), não deve conduzir ou trabalhar com máquinas nas 6 horas seguintes ou enquanto a sintomatologia de sonolência permanecer.

\section{Se antecedentes de reação de hipersensibilidade} ligeira

- O exame deve ser realizado;

- Pode ser efetuado contraste endovenoso, se benefício diagnóstico;

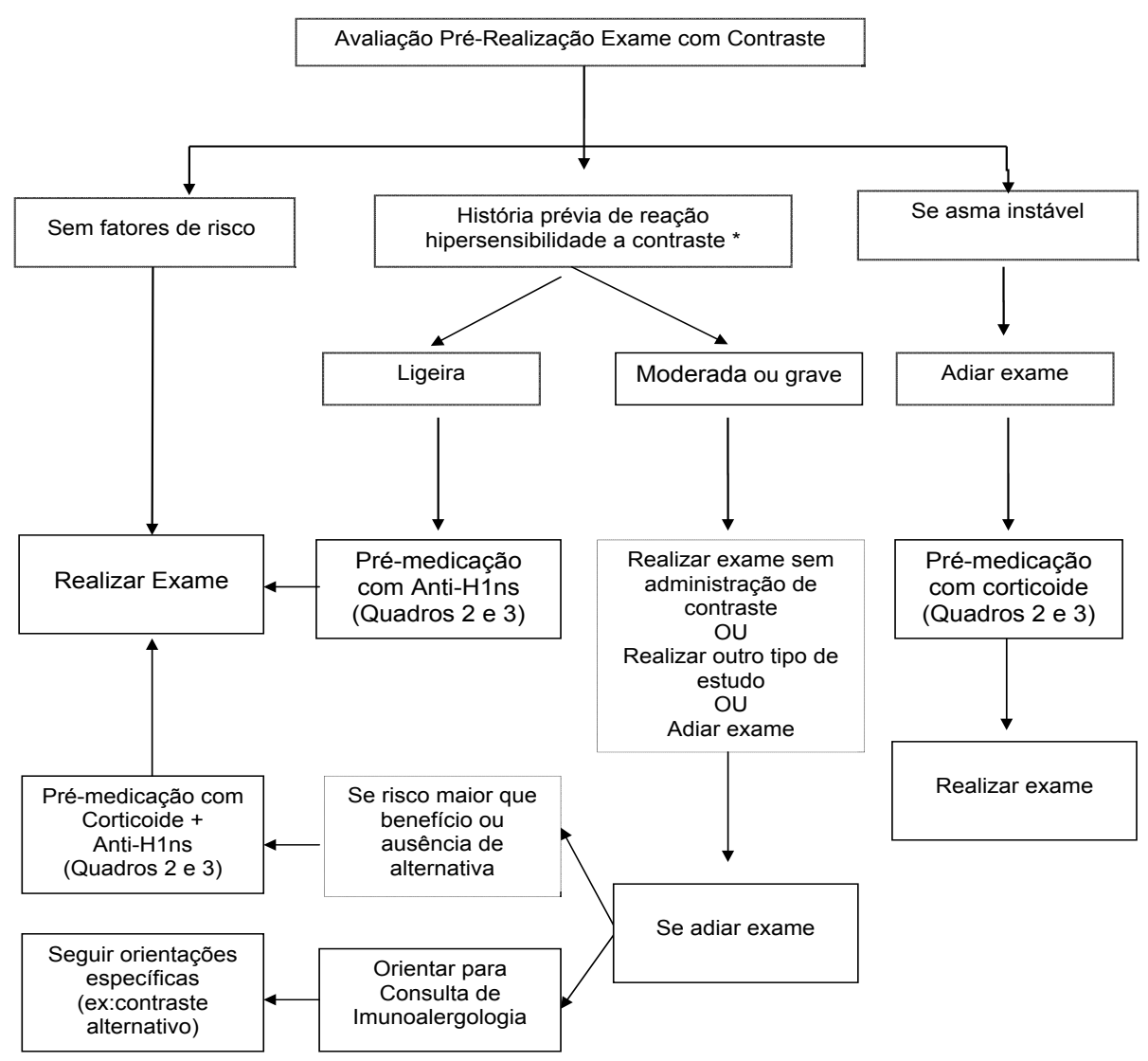

* lodo se indicação para TAC ou angiografia ou urografia e gadolínio se indicação para fazer ressonância magnética

Anti-HI ns- anti-histamínico $\mathrm{HI}$ não sedativo

Figura I. Abordagem do doente que vai realizar exame com meio de contraste 
- Se estudo efetuado com administração de meio de contraste idêntico ao associado a reação adversa prévia, ponderar anti-histamínico não sedativo per os 30 minutos antes da realização do exame (Quadros 2 e 3)

\section{Se antecedentes de reação e hipersensibilidade moderada a grave.}

- Considerar realizar o estudo de imagiologia sem meio de contraste;

- Considerar realizar outro tipo de estudo;

- Adiar o exame;

- E, se possível, orientar para a consulta de Imunoalergologia com vista a realizar estudo alergo- lógico e estabelecer um diagnóstico e orientações específicas;

- De acordo com o benefício, nomeadamente na ausência de alternativa, realizar o estudo imagiológico com administração de meio de contraste após medidas medicamentosas de "pré-medicação", de acordo com a situação clínica - corticoide + anti-histamínico não sedativo (Quadros 2 e 3).

- Nesta situação, considerar utilizar outro princípio farmacológico ativo de meio de contraste, diferente do utilizado no estudo prévio no qual ocorreu a reação de hipersensibilidade.

Quadro 2. Esquema de pré-medicação de acordo com factores de risco

\section{Esquema pré-medicação}

História prévia de reação de hipersensibilidade ligeira a produto de contraste

Anti-HI não sedativo 2. geração por via oral (PO) 30 minutos antes da administração do contraste

História prévia de reação de hipersensibilidade moderada a grave a produto de contraste e não pode adiar o exame esperando pela consulta de Imunoalergologia

Adultos e crianças com $\geq I 2$ anos

Prednisolona 50mg 13,7 e I hora antes PO ou metilprednisolona $32 \mathrm{mg}$ PO/ $2 \mathrm{~h}$ e $2 \mathrm{~h}$ antes

MAIS

Anti-HI não sedativo $2 .^{a}$ geração $\mathrm{PO} 2 \mathrm{~h}$ antes da administração do meio de contraste

Se doente não consegue fazer medicação oral optar por pré-medicação i.v:

Ex: Hidrocortisona $200 \mathrm{mg}$ iv, $12 \mathrm{~h}$ e $2 \mathrm{~h}$ antes contraste + Clemastina $2 \mathrm{mg}$ ev $2 \mathrm{~h}$ antes da administração do meio de contraste Se situações não programadas (doentes no SU):

Ex: Hidrocortisona $200 \mathrm{mg}$ ev a cada $4 \mathrm{~h} / 6 \mathrm{~h}$, até à realização do exame + Clemastina $2 \mathrm{mg}$ ev I hora antes de contraste.

\section{Crianças $\geq 6$ e $<12$ anos}

Prednisolona $0,5 \mathrm{mg} / \mathrm{kg}$ PO I3,7 e Ih antes ou metilprednisolona I mg/kg PO I $2 \mathrm{~h}$ e $2 \mathrm{~h}$ antes

MAIS

Anti-HI não sedativo $2{ }^{a}$ geração $\mathrm{PO} 2 \mathrm{~h}$ antes da administração do meio de contraste

\section{Crianças com $<6$ anos}

Ex: Betametasona $0,03 \mathrm{mg} / \mathrm{kg}$ PO (em geral corresponde a 2 gotas por $\mathrm{kg}$ ); 12 e $2 \mathrm{~h}$ antes da administração do produto de contraste MAIS

Anti-HI não sedativo $2{ }^{a}$ geração $\mathrm{PO} 2 \mathrm{~h}$ antes da administração do meio de contraste

\section{Asma instável}

Adultos e crianças com $>12$ anos

Ex: Prednisolona $50 \mathrm{mg}$ PO, $12 \mathrm{~h}$ e $2 \mathrm{~h}$ antes da administração de meio de contraste

\section{Crianças $\geq 6$ anos}

Ex: Prednisolona $0,5 \mathrm{mg} / \mathrm{kg}$ PO (até $50 \mathrm{mg}$ ); I $2 \mathrm{~h}$ e $2 \mathrm{~h}$ antes da administração do produto de contraste

\section{Crianças com $<6$ anos}

Ex: Betametasona $0,03 \mathrm{mg} / \mathrm{kg}$ PO (em geral corresponde a 2 gotas por $\mathrm{kg}$ ); I $2 \mathrm{~h}$ e $2 \mathrm{~h}$ antes da administração do produto de contraste 
Quadro 3. Fármacos que poderão ser utilizados como pré-medicação

\begin{tabular}{|c|c|c|c|}
\hline Nome & Formulação & Dose crianças & Dose adultos \\
\hline \multicolumn{4}{|c|}{ Anti-histamínicos } \\
\hline Fexofenadina & $S, C$ & $\begin{array}{l}\text { 6- } 12 \text { anos: } 30 \mathrm{mg} \\
>12 \text { anos: } 120-180 \mathrm{mg} \mid \mathrm{x} / \mathrm{dia}\end{array}$ & $120-180 \mathrm{mg}$ \\
\hline Cetirizina & S, C & $\begin{array}{l}2-5 \text { anos: } 2,5 \mathrm{mg} \\
6-1 \mathrm{I} \text { anos: } 5 \mathrm{mg} \\
>12 \text { anos: } 10 \mathrm{mg} / \mathrm{dia}\end{array}$ & $10 \mathrm{mg}$ \\
\hline Loratadina & S,C & $\begin{array}{l}2-11 \text { anos: } \\
\leq 30 \mathrm{~kg}: 5 \mathrm{mg} \\
>30 \mathrm{~kg}: 10 \mathrm{mg} \\
>12 \text { anos, > } 30 \mathrm{~kg}: 10 \mathrm{mg}\end{array}$ & $10 \mathrm{mg}$ \\
\hline Levocetirizina & $S, C$ & $\begin{array}{l}2-5 \text { anos: } 1,25 \mathrm{mg} \\
6-11 \text { anos: } 2,5 \mathrm{mg} \\
>12 \text { anos: } 5 \mathrm{mg}\end{array}$ & $5 \mathrm{mg}$ \\
\hline Desloratadina & $S, C$ & $\begin{array}{l}\text { I }-5 \text { anos: } 1,25 \mathrm{mg} \\
6-I I \text { anos: } 2,5 \mathrm{mg} \\
>12 \text { anos: } 5 \mathrm{mg}\end{array}$ & $5 \mathrm{mg}$ \\
\hline Ebastina & S,C & $\begin{array}{l}2-5 \text { anos: } 2,5 \mathrm{mg} / \mathrm{dia} \\
6-11 \text { anos: } 5 \mathrm{mg} / \mathrm{dia} \\
>12 \text { anos: } 10 \mathrm{mg} / \mathrm{dia}\end{array}$ & $10-20 \mathrm{mg}$ \\
\hline Rupatadina & $S, C$ & $\begin{array}{l}2-11 \text { anos, } \\
10-25 \mathrm{~kg}: 2,5 \mathrm{mg} \\
\geq 25 \mathrm{~kg}: 5 \mathrm{mg} \\
>12 \text { anos: } 10 \mathrm{mg}\end{array}$ & $10 \mathrm{mg}$ \\
\hline Bilastina & C & $>12$ anos: 20 mg/ dia & $20 \mathrm{mg}$ \\
\hline Clemastina & IM; IV & $0,025 \mathrm{mg} / \mathrm{kg}$ & $2 \mathrm{mg}$ \\
\hline \multicolumn{4}{|c|}{ Corticoides } \\
\hline Prednisolona & C, IV & $\begin{array}{l}0,5 \mathrm{mg} / \mathrm{kg} \mathrm{PO} \\
0,5 \mathrm{mg} / \mathrm{kg} \mathrm{IV}\end{array}$ & $\begin{array}{l}50 \mathrm{mg} \text { PO } \\
50 \mathrm{mg} \text { IV }\end{array}$ \\
\hline Metilprednisolona & C,IV & $\begin{array}{l}\mathrm{I} \mathrm{mg} / \mathrm{kg} \mathrm{PO} \\
0,5 \mathrm{mg} / \mathrm{kg} \text { IV }\end{array}$ & $\begin{array}{l}32 \mathrm{mg} \text { PO } \\
40 \mathrm{mg} \text { IV }\end{array}$ \\
\hline Hidrocortisona & IV & $4 \mathrm{mg} / \mathrm{kg}$ & $200 \mathrm{mg}$ \\
\hline Betametasona & $S$ & $\begin{array}{l}\text { < } 6 \text { anos: } \\
\text { Betametasona } 0,03 \mathrm{mg} / \mathrm{kg} \text { PO (em } \\
\text { geral corresponde a } 2 \text { gotas por } \mathrm{kg} \text { ); }\end{array}$ & \\
\hline Deflazacorte & $S, C$ & $\begin{array}{l}<6 \text { anos } \\
1 \mathrm{mg} / \mathrm{kg}(=1 \text { gota por kg) }\end{array}$ & $30 \mathrm{mg}$ \\
\hline
\end{tabular}

S - solução oral; C - comprimido; PO - por via oral; IV - via intravenosa 


\section{Se asma instável}

No caso de o utente referir diagnóstico médico de asma com sintomas não controlados como pieira ou broncospasmo, dispneia e baixa tolerância ao esforço:

- Considerar realizar o estudo de imagiologia sem meio de contraste;

- Se necessário realizar estudo de imagiologia com meio de contraste, este deve ser adiado e programada pré-medicação - corticoide; neste caso particular é importante a realização do corticóide como anti-edematoso (Quadros 2 e 3).

\section{Se História de reação alérgica a outros alergénios}

Seguir as indicações gerais especificadas; esta situação, por si, não requer a realização de pré-medicação.

\section{CONSIDERAÇÕES SOBRE REAÇÕES QUIMIOTÓXICAS}

Com o objetivo de minimizar as reações quimiotóxicas a meio de contraste em imagiologia, devemos:

- Administrar contraste em estudo de imagiologia apenas se houver benefício diagnóstico;

- Cumprir os cuidados de acondicionamento e manuseamento referidos pelo fabricante;

- No caso dos meios de contraste com IODO, utilizar meios de contraste iso-osmolares ou de baixa osmolaridade;

- Administrar a mínima dose / volume necessário e o menor fluxo de administração possível, de acordo com a indicação clínica para o exame;

- No caso de estudo com meio de contraste com IODO, na ponderação de volume e fluxo de administração deve também atender-se ao perfil do paciente, nomeadamente à presença de patologia cardíaca e risco de sobrecarga hídrica;

- Perceber que a ansiedade prévia e associada à realização de estudo de imagiologia com meio de contraste pode, por si só, condicionar a ocorrência de sintomatologia idêntica a uma verdadeira reação adversa a meio de contraste.

\section{TRATAMENTO DAS REAÇÕES ADVERSAS AGUDAS NÃO RENAIS A MEIOS DE CONTRASTE}

Qualquer utente pode desenvolver, em qualquer estudo de imagiologia com meio de contraste uma reação adversa aguda, que deve ser prontamente identificada e tratada. O doente deve ser orientado por forma a obter-se um diagnóstico clínico ou clínico-laboratorial com implicações em situações futuras de exames de imagiologia.

A abordagem das reações adversas agudas não renais a meios de contraste está sumarizada na Figura 2.

Há um conjunto de medidas universais que devem ser tomadas em todas as reações adversas e que incluem a suspensão da administração do contraste, a colocação de oxigénio e a monitorização:

- Eliminar o factor desencadeante é fundamental, correspondendo neste caso à interrupção da administração do produto de contraste;

- Todos os doentes devem ser colocados numa posição confortável. Podem preferir estar sentados, já que lhes vai facilitar a respiração. $O$ decúbito dorsal, com elevação dos membros inferiores, é útil nos doentes hipotensos e quando pensamos estar a ocorrer uma reação vagal. Enquanto tiver sensação de desmaio, o doente não se deve sentar nem levantar. O doente deve ser colocado em local onde possa ser despido facilmente, de modo a observar-se a progressão das lesões cutâneas;

- A monitorização deve incluir sempre pelo menos a frequência respiratória, oximetria de pulso, pressão arterial não invasiva e frequência cardíaca;

- Deve ser colocado oxigénio por máscara facial (máscara de alta concentração) até à reavaliação da situação clínica. 


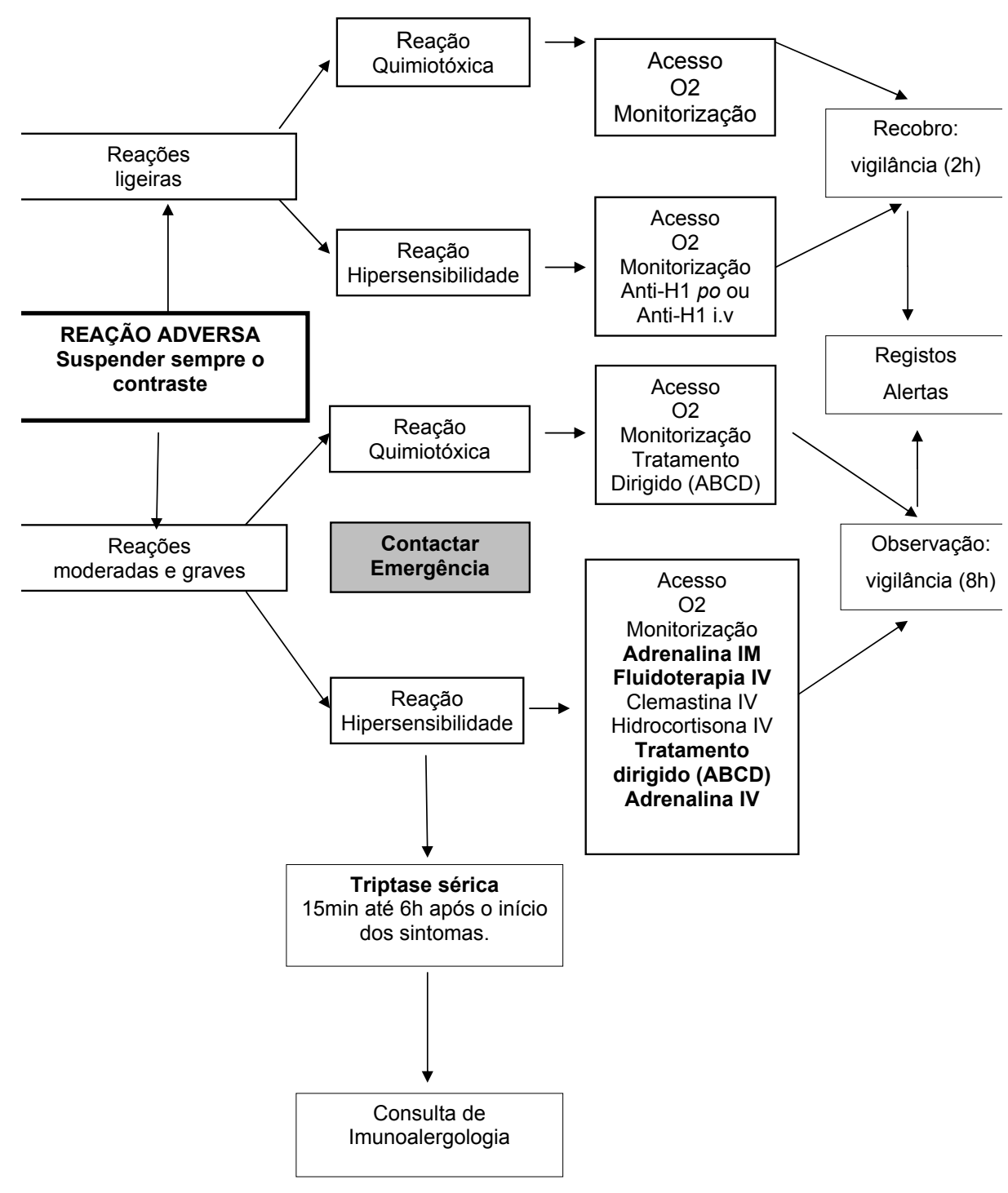

Figura 2. Tratamento das reações de hipersensibilidade a meios de contraste.

Perante uma reação ligeira devem ser tomadas apenas as medidas universais e se houver resolução dos sintomas, a vigilância do utente deve ser mantida, no serviço de imagiologia, durante pelo menos 30 minutos depois da resolução dos mesmos.

Se a suspeita for de reação de hipersensibilidade ligeira pode usar-se um fármaco anti-histamínico, preferencialmente não sedativo de segunda geração. A vigilância deve ser mantida durante pelo menos 30 minutos depois da resolução dos sintomas.

Perante uma reação moderada ou grave deve ser ativada de imediato a equipa de emergência interna. Se a suspeita for de reação de hipersensibilidade, o médico de serviço de Imagiologia deve administrar de imediato adrenalina IM (ver Quadro 4). Até à chegada da equipa de emergência interna devem os profissionais presentes 
tomar as medidas de suporte básico de vida adequadas ao caso clínico.

\section{Tratamento das reações quimiotóxicas}

O tratamento destas reações é sintomático e dirigido ao tipo de reação.

Como a maior parte das vezes o que acontece é uma reação ligeira, está indicado manter vigilância do doente no serviço de imagiologia até 30 minutos após a resolução dos sintomas.

Se a reação for moderada ou grave deve envolver-se a equipa de emergência interna, que fará o tratamento dirigido. Neste caso a vigilância deverá ser estendida até às 8 horas, em regime de internamento.

\section{Tratamento das reações de hipersensibilidade}

\section{Ligeiras}

Perante uma reação ligeira devem ser tomadas as medidas universais, monitorização e vigilância já descritas. Os fármacos anti-histamínicos indicados são não sedativos (Quadro 3) ou no caso de impossibilidade de via oral pode usar-se por exemplo a clemastina (a dose no adulto são os $2 \mathrm{mg}$ IV ou IM; na criança a dose correta é de $0,025 \mathrm{mg} / \mathrm{kg} /$ dose IM. ou EV (até um máximo de $2 \mathrm{mg}$ ) (ver Quadro 4).

\section{Moderadas ou graves}

É provável estarmos perante uma reação de hipersensibilidade moderada ou grave quando um doente

Quadro 4. Fármacos utlizados no tratamento das reações de hipersensibilidade

\section{ADRENALINA \\ (administrar sempre por via IM - dose por peso - 0,0I mg/kg/dose)}

Adulto e criança com mais de $\mathbf{I} 2$ anos-administrar uma dose inicial de $0,5 \mathrm{mg}$ de adrenalina $\mathrm{IM}(0,5 \mathrm{ml}$ de I ampola de I mg/ml = $=0,5 \mathrm{mg}=500 \mathrm{mcg}$ ). Podem ser administradas doses adicionais com intervalos de cerca de 5 minutos até 3 doses.

Crianças com idade entre 6 e 12 anos-administrar uma dose inicial de 0,3 mg de adrenalina IM $(0,3 \mathrm{ml}$ de I ampola de I mg/ml = $=0,3 \mathrm{mg}=300 \mathrm{mcg})$.

Crianças com menos de 6 anos - administrar uma dose inicial de 0,15 mg de adrenalina IM $(0,15 \mathrm{ml} \mathrm{de} \mathrm{I} \mathrm{ampola} \mathrm{de} \mathrm{I} \mathrm{mg/ml} \mathrm{=}$ $=0,15 \mathrm{mg}=150 \mathrm{mcg})$.

\section{Adrenalina IV}

(apenas em caso de ausência de resposta a adrenalina IM e por médico com experiência na sua utilização) (diluir uma ampola de adrenalina $(\mathrm{Img} / \mathrm{ml})$ até $100 \mathrm{ml}$ de soro fisiológico - fica uma concentração de I0ug/ml)

Adulto: Bólus de 20 a 50 ug repetidos a cada I-2 minutos, se necessário; perfusão de $0, \mathrm{I}-\mathrm{l}$ ug/kg/min, pode ser usada a mesma diluição Pediatria: infusão contínua $0, \mathrm{I}$ - l ug/ $/ \mathrm{kg} / \mathrm{min}$. Bólus IV não aplicável.

\section{Fluidos}

Adulto - bólus rápido de fluidos IV (500-1000 ml de cristaloide), se necessário administrar doses adicionais. Crianças - bólus de fluidos IV (20 ml/kg de cristaloides).

\section{Outros fármacos}

Clemastina - 0,025 mg/kg/dose EV/IM (máximo - $2 \mathrm{mg}$ )

Hidrocortisona - adulto - $200 \mathrm{mg}$; crianças - $4 \mathrm{mg} / \mathrm{kg}$

Glucagon (resistência à adrenalina nos doentes beta-bloqueados) - bólus de $30 \mathrm{ug} / \mathrm{kg} / \mathrm{dose} \mathrm{IV}$ até ao máximo de I mg (pode ser repetido a cada $5 \mathrm{~min}$ ).

Perfusão contínua $5-15 \mathrm{ug} / \mathrm{min}$ (diluir uma ampola até $100 \mathrm{ml}$ de soro fisiológico $(10 \mathrm{ug} / \mathrm{ml})$ ). 
desenvolve doença súbita e inesperada (habitualmente nos minutos que se seguem à administração de produto de contraste), com alterações cutâneas de progressão rápida e compromisso potencialmente fatal da via aérea e/ou respiratório e/ou circulatório.

A reação alérgica, sendo um tipo específico e potencialmente fatal de reação adversa, tem uma abordagem diferente. Esta abordagem deve ser adequada à gravidade clínica do doente. Este algoritmo de atuação é baseado nas recomendações do Conselho Europeu de Ressuscitação (ERC).

\section{Abordagem $A B C D E$}

$A$ metodologia $A B C D E$ de reconhecimento e abordagem de qualquer doente crítico é a que deve ser utilizada para estas situações.

Os problemas da via aérea ( $A$ - airway) podem corresponder a edema das vias aéreas e traduzir-se por rouquidão ou estridor e podem evoluir até à obstrução completa e paragem cardiorrespiratória. A obstrução da via aérea pode acontecer de forma rápida. Os sinais de alerta são o edema da língua e dos lábios e a rouquidão. $O$ envolvimento precoce de um perito da via aérea (anestesiologista experiente; intensivista) é obrigatório no tratamento destes doentes. É necessário ponderar a intubação traqueal precoce; qualquer atraso pode tornar a intubação extremamente difícil. Pode ser necessária uma via aérea cirúrgica, caso a intubação traqueal não seja possível.

Os problemas da respiração (B - breathing) podem traduzir-se em dispneia, pieira, sibilos, cianose e confusão, traduzindo frequentemente broncoconstrição e insuficiência respiratória.

Os problemas circulatórios ( $\mathrm{C}$ - circulation) podem ir da hipotensão à paragem cardíaca. Os problemas circulatórios (muitas vezes referidos como choque anafilático) podem ser causados por depressão miocárdica direta ou vasodilatação e aumento da permeabilidade capilar.

Os problemas da via aérea, respiração e circulação, já referidos, podem alterar o estado neurológico do doente (D - disability) por diminuição da perfusão cerebral. Pode haver confusão, agitação e perda de consciência.
Os problemas da exposição ( $E$ - exposure) significam a necessidade de controlo da exposição, nomeadamente com controlo da temperatura corporal.

\section{Adrenalina}

De notar que perante a suspeita de reação moderada ou grave de hipersensibilidade o fármaco de eleição é a adrenalina ${ }^{5,6}$

Não existem contraindicações absolutas para tratamento com adrenalina intramuscular numa situação de anafilaxia. Os efeitos adversos são muito raros quando são injetadas as doses corretas por via intramuscular (IM). As vias subcutâneas ou inalatórias não são recomendadas.

No adulto e na criança commais de 12 anos, administrar uma dose inicial de $0,5 \mathrm{mg}$ de adrenalina IM $(0,5 \mathrm{ml}$ de ampola I mg/ml (I:1000 adrenalina) $=0,5 \mathrm{mg}=500 \mathrm{mcg}$ ). Podem ser administradas doses adicionais com intervalos de cerca de 5 minutos, em função da resposta do doente, até 3 administrações.

Nas crianças com idade entre 6 e 12 anos a dose de adrenalina é de $0,3 \mathrm{mg}$ de IM $(0,3 \mathrm{ml}$ de ampola $\mathrm{I} \mathrm{mg} / \mathrm{ml}$ $(\mathrm{l}: 1000$ adrenalina $)=0,3 \mathrm{mg}=300 \mathrm{mcg})$. Nas crianças com menos de 6 anos a dose de adrenalina é de $0,15 \mathrm{mg}$ de IM $(0,15 \mathrm{ml}$ de ampola I mg/ml (I:1000 adrenalina $)=0,15 \mathrm{mg}$ $=150 \mathrm{mcg}$ ) (Quadro 4).

O melhor local para a injeção IM é a porção anterolateral do terço médio da coxa. A agulha a utilizar na injeção tem de ser suficientemente comprida para assegurar que a adrenalina é injetada no músculo (2IG-cor verde, com $4 \mathrm{~cm}$, também conhecida por agulha intramuscular).

A adrenalina IV só deve ser administrada por profissionais experientes na utilização e titulação de vasopressores na prática clínica (por exemplo, anestesiologistas ou intensivistas) ${ }^{7}$. Em doentes com circulação espontânea, a adrenalina intravenosa pode provocar hipertensão potencialmente fatal, taquicardia, arritmias e isquemia do miocárdio. Não administrar a solução de adrenalina I:I.000 por via IV sem diluição prévia apropriada (diluir $\mathrm{I} \mathrm{mg}$ até $100 \mathrm{ml}$ de soro fisiológico, pode usar esta diluição para bólus ou para infusão). Titular a adrenalina IV 
em bólus de 20 a $50 \mathrm{mcg}$ ( 2 a $5 \mathrm{ml}$ da diluição prévia proposta) em função da resposta. Nas crianças não fazer adrenalina IV em bólus. Se forem necessárias doses repetidas, estabelecer perfusão IV de adrenalina usando os quadros que existem para o efeito $(1$ a $10 \mathrm{mcg} / \mathrm{min}$ é a dose habitual, o que corresponde a 6 a $60 \mathrm{ml} / \mathrm{h}$ da diluição prévia, titulando para o efeito pretendido).

A adrenalina continua a ser o vasopressor de primeira linha para o tratamento de reações alérgicas. Ponderar outros vasopressores e inotrópicos (noradrenalina, vasopressina, terlipressina) quando a reanimação inicial com adrenalina e fluido não é bem-sucedida. Estes fármacos só devem ser usados em contextos especializados (bloco, unidades de cuidados intensivos), onde há experiência na sua utilização. $O$ glucagon pode ser útil para tratar reações alérgicas em doentes medicados com betabloqueadores.

\section{Fluidoterapia}

A fluidoterapia atempada é um dos factores determinantes para evitar e corrigir a hipotensão/choque distributivo que poderá conduzir a paragem cardiorrespiratória. Deve-se administrar um bólus rápido de fluídos IV (500-1000 ml de um cristaloide no adulto), monitorizar a resposta e se necessário administrar doses adicionais. Nas crianças está recomendado um bólus de fluidos cristalóides de $20 \mathrm{ml} / \mathrm{kg}$. Neste contexto entende-se por cristaloide o soro polieletrolítico para uso endovenoso, isotónico e sem glicose, como por exemplo o Poli ${ }^{\circledR}$ ou o Plasma-Lyte ${ }^{\circledR}$.

\section{Outros fármacos}

A administração de anti-histamínicos, embora reduza os sintomas, não é life saving e nunca deve atrasar a administração de adrenalina intramuscular. $O$ fármaco anti-histamínico sugerido é a clemastina. A dose no adulto são os $2 \mathrm{mg}$ IV ou IM. Na criança a dose correta é de $0,025 \mathrm{mg} / \mathrm{kg} /$ dose IM ou EV (até um máximo de $2 \mathrm{mg}$ ).

Há pouca evidência que suporte a utilização por rotina de anti-histamínicos $\mathrm{H} 2$ (p. ex. ranitidina, cimetidina) para o tratamento destas situações.
A administração de corticoide serve essencialmente para a prevenção de reações bifásicas (recrudescimento da reação alérgica após 6 a 8 h) e não é também life saving e nunca deve atrasar a administração de adrenalina intramuscular. Há pouca evidência sobre qual a dose ideal por exemplo de hidrocortisona, sugerindo-se no adulto $200 \mathrm{mg}$ IV e na criança $4 \mathrm{mg} / \mathrm{kg}$ IV lento.

\section{Paragem cardiorrespiratória}

Em caso de paragem cardiorrespiratória devem iniciar-se de imediato as medidas de suporte avançado de vida, de acordo com as mais recentes guidelines do ERC.

\section{PROCEDIMENTOS LABORATORIAIS}

O diagnóstico de reações de hipersensibilidade é clínico e os meios complementares são apenas auxiliares. Existem vários diagnósticos diferenciais que devem ser considerados de acordo com a gravidade das manifestações clínicas e excluídos quando necessário, nunca atrasando a intervenção de tratamento.

Em todas as reações de hipersensibilidade moderadas a graves a produto de contraste deve realizar-se uma coIheita de sangue para quantificação dos níveis de triptase sérica. A colheita deve ser o mais precoce possível entre 15 minutos a 6 horas após o início dos sintomas.

Em todas as reações de hipersensibilidade moderadas a graves com produto de contraste deve ser pedida consulta de Imunoalergologia. Na consulta de Imunoalergologia vão ser interpretados os resultados da triptase sérica. Os aumentos de triptase sérica apoiam o diagnóstico de hipersensibilidade mediada por IgE, mas, se normais, não excluem uma reação anafilática. Em caso de elevação do valor de triptase sérica deverá ser feita nova colheita após a resolução dos sintomas, no follow-up do doente, em consulta de Imunoalergologia. Outros meios complementares de diagnóstico serão realizados durante o seguimento em consulta de Imunoalergologia. 


\section{ALTA E REFERENCIAÇÃo}

Os doentes com reação de hipersensibilidade moderada ou grave devem ser tratados e observados durante pelo menos 8 horas num serviço com capacidade para tratar estas situações ${ }^{8}$. Desconhece-se a incidência exata de reações bifásicas que poderiam justificar uma vigilância mais prolongada. Não há uma forma fidedigna de prever quem vai sofrer uma reação bifásica. Assim, é importante que as decisões sobre a alta sejam tomadas para cada doente individualmente por um médico experiente. Não há indicação para a manutenção de tratamento em ambulatório se todos os sintomas resolveram.

Antes da alta todos os doentes devem:

- Ser informados sobre a natureza da reação e sobre a necessidade de continuar a investigação clínica. O doente será informado ainda de que deve evitar o meio de contraste até ir à consulta de Imunoalergologia;

- Receber instruções claras para regressarem ao hospital se os sintomas ressurgirem;

- Ser referenciados a um especialista em Imunoalergologia.

Assim, devem ser referenciados para a consulta de Imunoalergologia os seguintes doentes:

- Com risco acrescido para reação de hipersensibilidade a contraste (suspeita de reação prévia moderada ou grave ao produto de contraste). Referenciação pelo médico Imagiologista ou pelo médico que requisita o exame com contraste;

- Que tiveram uma reação de hipersensibilidade moderada ou grave ao produto de contraste.

\section{REGISTOS E ALERTAS}

Sempre que ocorrer uma reação adversa aguda não nefrotóxica a produto de contraste deve ser feito um registo clínico ${ }^{8}$. Este registo deve conter a descrição das manifestações clínicas, terapêutica realizada e tempo até à resolução dos sintomas.

Deve ainda ser feito o registo da reação adversa no site da INFARMED (em plataforma própria), sendo que sempre que necessário deverá socorrer-se de informação do enfermeiro ou técnico.

A inserção da informação de reação de hipersensibilidade a produto de contraste no processo clínico/eletrónico do doente sob a forma de alerta é fundamental para prevenir futuras administrações e reações.

\section{Agradecimentos}

Aos Coordenadores do Grupo de Interesse de Alergia a Fármacos da Sociedade Portuguesa de Imunoalergologia.

Às colegas Dr. Ana Luísa Geraldes e Dr. ${ }^{a}$ Joana Caiado, pela revisão do documento.

\section{Conflitos de interesse}

Nenhuns.

\section{Contacto:}

Cristina Lopes

E-mail: cristina.lopes.abreu@gmail.com

\section{REFERÊNCIAS}

I. Rosado Ingelmo A, Dona Diaz I, Cabanas Moreno R, Moya Quesada MC, Garcia-Aviles C, Garcia Nunez I, et al. Clinical practice guidelines for diagnosis and management of hypersensitivity reactions to contrast media. J Investig Allergol Clin Immunol. 2016; 26:144-55;

2. Media ACoRCoDaC. Manual on contrast media version I0.32017. In: Radiology ACo, ed: American College of Radiology 2017: http:// www.acr.org

3. Salas M, Gomez F, Fernandez TD, Dona I, Aranda A, Ariza A, et al. Diagnosis of immediate hypersensitivity reactions to radiocontrast media. Allergy. 2013;68:1203-6.

4. Laroche D, Aimone-Gastin I, Dubois F, Huet H, Gerard P, Vergnaud $M C$, et al. Mechanisms of severe, immediate reactions to iodinated contrast material. Radiology 1998;209:183-90.

5. Direção Geral da Saúde. Anafilaxia: Abordagem clínica. 2014, Norma da Direção-Geral de Saúde N. ${ }^{\circ} 014 / 2012$ de 16/12/2012 


\section{Cristina Lopes, Catarina Tavares, Inês Rolla, Ernestina Gomes}

atualizada a $18 / / 2 / 20 \mid 4$. Disponivel em em www.dgs.pt a 10.01.2018

6. Simons FE, Ebisawa M, Sanchez-Borges M, Thong BY, Worm M, Tanno LK, et al. 2015 update of the evidence base: World Allergy Organization anaphylaxis guidelines. World Allergy Organ J 20I5;8:32.

7. Truhlar A, Deakin CD, Soar J, Khalifa GE, Alfonzo A, Bierens JJ, et al. European Resuscitation Council Guidelines for Resuscitation
2015: Section 4. Cardiac arrest in special circumstances. Resuscitation 2015;95:148-20I.

8. Direção-Geral da Saúde. Anafilaxia: Registo e Encaminhamento 2014, Norma da Direção-Geral de Saúde N. $014 / 2012$ de 16/I2/2012 atualizada a 18/12/2014. Disponivel em em www.dgs. pt a 10.01.2018. 